\title{
Correlações genéticas e análise de trilha para número de frutos comerciais por planta em mamoeiro
}

\author{
Eder Jorge de Oliveira ${ }^{(1)}$, Diego Souza de Lima ${ }^{(2)}$, Rangel Sales Lucena(2), Tiago Borges Nunes Motta(2) \\ e Jorge Luiz Loyola Dantas(1)
}

\begin{abstract}
(1)Embrapa Mandioca e Fruticultura Tropical, Rua da Embrapa, s/no, Caixa Postal 007, CEP 44380-000 Cruz das Almas, BA. E-mail: eder@cnpmf.embrapa.br, loyola@cnpmf.embrapa.br (2)Universidade Federal do Recôncavo da Bahia, Campus Universitário de Cruz das Almas, CEP 44380-000 Cruz das Almas, BA. E-mail: diegodelima10@yahoo.com.br, rangel_lucena@yahoo.com.br, tico_motta@hotmail.com
\end{abstract}

Resumo - O objetivo deste trabalho foi verificar como o número de frutos comerciais por planta (NFr) se relaciona com 16 características morfológicas e agronômicas do mamoeiro (Carica papaya L.). O experimento foi realizado no ano agrícola de 2008/2009, utilizando o delineamento experimental de blocos aumentados. Foram avaliados 19 genótipos não comuns (acessos de germoplasma) e 3 comuns (variedades e híbridos), em 10 repetições. As correlações entre NFr e as características morfoagronômicas do mamoeiro foram desdobradas em efeitos diretos e indiretos. Observou-se alta variabilidade nas características avaliadas. As estimativas das correlações genotípicas foram maiores do que as fenotípicas, para todas as características. O NFr foi positivo e significativamente correlacionado com número de frutos por axila (NFrAx); e negativamente com diâmetro da cavidade do fruto, espessura da polpa, peso, comprimento ( $\mathrm{CFr}$ ) e diâmetro do fruto. Entretanto, a análise de trilha demonstrou que, entre essas características, NFrAx e CFr possuem maior efeito direto sobre NFr. Além disso, as características altura da planta, número de flores por pedúnculo e largura da folha são de mais fácil mensuração e manifestam-se antes da produção de frutos, o que as torna mais indicadas para a seleção indireta em programas de melhoramento genético dessa cultura.

Termos para indexação: Carica papaya, características morfoagronômicas, componentes da produção, melhoramento genético, seleção indireta.

\section{Genetic correlation and path analysis for the number of commercial fruit per plant in papaya}

\begin{abstract}
The objective of this work was to determine how the number of commercial fruit per plant ( $\mathrm{CFrP})$ is related to 16 morphological and agronomical traits in papaya (Carica papaya L.). The experiment was carried out in the 2008/2009-crop season, using an augmented randomized block design. Nineteen non-common (germplasm accessions) and three common genotypes (varieties and hybrids) were evaluated with ten repetitions. Correlations between CFrP and morpho-agronomic traits were outspread in direct and indirect effects. High variability was found for the evaluated traits. The estimates of genetic correlations were higher than phenotypic correlations for all characters. CFrP was positively and significantly correlated with number of fruits per axil (NFAx) and negatively with the internal diameter of the cavity of the fruit, pulp thickness, fruit weight, fruit length (FrL) and fruit diameter. However, path analysis showed that, among this traits, NFAx and FrL have more direct effect on CFrP. Furthermore, plant height, number of flowers per peduncle and leaf width are more easily measured and are expressed before fruit production, making them more suitable for indirect selection in breeding programs of this crop.
\end{abstract}

Index terms: Carica papaya, morpho-agronomic traits, yield components, breeding, indirect plant selection.

\section{Introdução}

Pesquisas desenvolvidas com a cultura do mamoeiro (Carica papaya L.) são relevantes para o Brasil, pois o país é um dos maiores produtores mundiais dessa fruta. A produção anual chega a alcançar aproximadamente 1,6 milhão de toneladas e abrange $25 \%$ do mercado mundial (Associação Brasileira dos Exportadores de Papaya, 2006).
Há pequena disponibilidade de cultivares para uso na cultura do mamoeiro. Esse fato torna seu cultivo mais vulnerável a doenças, pragas e variações edafoclimáticas, o que prejudica a sustentabilidade desse agronegócio. Dessa forma, o melhoramento genético pode contribuir para aumentar a variabilidade genética dos cultivos de mamoeiro por meio do desenvolvimento de novos genótipos, com resistência às principais pragas, com atributos agronômicos e

Pesq. agropec. bras., Brasília, v.45, n.8, p.855-862, ago. 2010 
de qualidade de frutos que atendam às exigências do consumidor. Para isso, tanto o conhecimento sobre a variabilidade genética disponível na espécie como o entendimento de quanto determinadas características relacionam-se para a formação de genótipos com tipos agronômicos desejáveis são pré-requisitos fundamentais para o sucesso dos programas de melhoramento genético.

A importância da correlação entre características reside na possibilidade de se avaliar o quanto a alteração em um caráter pode afetar os demais. Se a seleção de um caráter é dificultada pela baixa herdabilidade ou por problemas de mensuração e identificação, esse tipo de conhecimento é importante nas diferentes etapas dos programas de melhoramento (Cruz et al., 2004). Como a maioria dos programas de melhoramento leva em consideração muitas características simultaneamente, o entendimento da associação genética entre elas pode contribuir para a escolha dos procedimentos de seleção mais apropriados para maximizar o ganho genético por geração (Santos \& Vencovsky, 1986).

No entanto, em muitos casos, a correlação fenotípica pode ter pouca aplicabilidade, e pode, inclusive, induzir o melhorista a erros (Chaudhary et al., 1973). É preciso distinguir, nos diferentes fenótipos, o que se deve a causas genéticas e a causas ambientais, para melhorar a precisão dos resultados (Almeida, 1988). No caso do mamoeiro, a identificação de características de alta herdabilidade e de fácil aferição, correlacionadas a atributos relativos à produtividade e à qualidade de frutos - que geralmente são controlados por vários genes e sofrem forte efeito ambiental -, permite ao melhorista obter progressos mais rápidos em relação ao uso de seleção direta, como observado em outras espécies (Santos \& Vencovsky, 1986; Carvalho et al., 1999, 2004).

As análises de correlação entre características produtivas e componentes de produção são essenciais na determinação dos critérios de seleção; todavia, a magnitude e o valor das correlações não são suficientes para esclarecer as relações entre as variáveis estudadas, pois pode não haver uma relação real de causa e efeito. Dessa maneira, um alto ou baixo coeficiente de correlação pode ser o resultado do efeito de outras variáveis, sem revelar a exata importância relativa dos efeitos diretos e indiretos desses fatores (Cruz et al., 2004). A fim de reduzir tais problemas, Wright $(1921,1923)$ propôs a metodologia de análise de trilha, que desdobra as correlações estimadas em efeitos diretos e indiretos de caracteres sobre uma variável básica.

O sucesso da análise de trilha reside basicamente na formulação do relacionamento causa-efeito entre as variáveis (Schuster, 1996), bem como na experiência do pesquisador em desdobrar as correlações para as características de maior importância agronômica (Cruz et al., 2004). Recentemente, essa técnica tem sido amplamente utilizada no estudo de diversas fruteiras (Reynolds \& Wardle, 1993; Okut \& Akca, 1995; Sirisenaa \& Senanayake, 2000; Negreiros et al., 2007). Porém, ainda são escassos os trabalhos dessa natureza envolvendo a cultura do mamoeiro.

O objetivo deste trabalho foi obter estimativas de correlações entre diversas características morfológicas e agronômicas do mamoeiro, bem como analisar a relação entre essas características e seus desdobramentos em efeitos diretos e indiretos, para nortear o melhoramento genético na obtenção de plantas de mamoeiro com maior número de frutos.

\section{Material e Métodos}

O experimento foi realizado na Embrapa Mandioca e Fruticultura Tropical, localizada no Município de Cruz das Almas, BA, na safra de 2008/2009 (1240'39"S e $39^{\circ} 06^{\prime} 23^{\prime \prime} \mathrm{W}$, a $220 \mathrm{~m}$ de altitude). A temperatura média anual é de $24,5^{\circ} \mathrm{C}$, a precipitação média anual de $1.240 \mathrm{~mm}$ e a umidade relativa média do ar de $82 \%$.

Foram avaliados 19 acessos de mamoeiro pertencentes ao Banco Ativo de Germoplasma da Embrapa Mandioca e Fruticultura Tropical (BAG-Mamão): CMF005, CMF008, CMF010, CMF014, CMF018, CMF020, CMF023, CMF028, CMF040, CMF046, CMF054, CMF055, CMF058, CMF065, CMF067, CMF068, CMF152, CMF232 e CMF234. Além deles, foram também avaliadas as variedades Golden e Sunrise Solo, bem como o híbrido Calimosa.

Utilizou-se o delineamento experimental de blocos aumentados, com 19 genótipos não comuns, três comuns, e dez repetições. O plantio foi realizado no espaçamento de $2 \times 2 \mathrm{~m}$, tendo-se seguido todos os tratos culturais recomendados para a cultura (Martins $\&$ Costa, 2003).

A avaliação das plantas foi iniciada oito meses após o plantio, tendo-se observado as seguintes características: 
comprimento do pecíolo da folha $(\mathrm{CPFo}, \mathrm{cm})$; comprimento da folha madura $(\mathrm{CFo}, \mathrm{cm})$; largura da folha madura (LFo, cm); altura da planta (APl, cm); número de flores por pedúnculo (NFIPed); número de frutos por axila (NFrAx); número de frutos comerciais por planta (NFr); firmeza do fruto (FFr, libras $\mathrm{cm}^{-2}$ ), utilizando penetrômetro manual modelo FT 327 (McCormick Fruit Tech, Yakima, EUA); massa do fruto (PFr, g); comprimento do fruto (CFr, cm); diâmetro do fruto (DFr, cm); diâmetro da cavidade do fruto (DCaFr, cm); espessura da polpa, em mm (EPol); acidez total titulável (\% de ácido cítrico), por titulação com NaOH 0,1 M (ATT); sólidos solúveis totais (SST), medido em ${ }^{\circ}$ Brix, com auxílio de um refratômetro manual; e razão entre AAT e SST (Ratio).

As medidas das características relacionadas às flores e frutos foram realizadas aos 12 meses após o plantio. Foram avaliadas três folhas por planta em quatro plantas. $\mathrm{Na}$ análise, foram colhidos aleatoriamente 12 frutos, 3 por planta, no estágio 1 de amadurecimento, quando o fruto apresenta até $15 \%$ da superfície amarela. Entretanto, as análises físico-químicas foram realizadas quando os frutos atingiram o estágio 4 , quando o fruto apresenta 50 a $75 \%$ da superfície amarela.

Os dados obtidos foram submetidos à análise de variância, de acordo com o modelo; $Y_{i j}=\mu+\tau_{i}+B_{j}+\varepsilon_{j}$, em que: $Y_{\mathrm{ij}}$ é o valor da característica para a i-ésima testemunha no j-ésimo bloco; $\mu$ é a média geral do experimento; $\tau_{\mathrm{i}}$ é o efeito do i-ésimo tratamento, que pode ser decomposto em $\mathrm{T}_{\mathrm{i}}=$ efeito da i-ésima testemunha, com $\mathrm{i}=1,2, \ldots . \mathrm{t}$ e $\mathrm{G}_{\mathrm{i}}^{\mathrm{j}}=$ efeito do i-ésimo genótipo, com $\mathrm{i}=1,2, \ldots . \mathrm{g}_{\mathrm{j}} ; \mathrm{B}_{\mathrm{j}}$ é o efeito do j-ésimo bloco; $\varepsilon_{\mathrm{ij}}$ é o erro aleatório.

Em seguida, foram estimados os coeficientes de correlação genotípica $\left(\mathrm{r}_{\mathrm{g}}\right)$, fenotípica $\left(\mathrm{r}_{\mathrm{f}}\right)$ e de ambiente $\left(\mathrm{r}_{\mathrm{e}}\right)$, com uso de análises de covariâncias, tendo-se combinado os dados das 16 características em todas as formas possíveis. As correlações foram desdobradas em efeitos diretos e indiretos das características de importância agronômica para o mamoeiro (variáveis independentes) sobre o número de frutos por planta (variável básica), por meio da análise de trilha (Wright, 1921, 1923; Cruz et al., 2004).

A análise de colinearidade entre as variáveis independentes foi verificada pela análise dos autovalores da matriz ( $\left.\mathrm{X}^{\prime} \mathrm{X}\right)$, conforme descrito por Carvalho et al. (2002), em que o grau de multicolinearidade foi estabelecido com base no número de condição (NC), que é a razão entre o maior e o menor autovalor da matriz (Montgomery \& Peck, 1982). Depois de constatado forte grau de multicolinearidade entre algumas variáveis, utilizou-se a metodologia de Carvalho (1995), denominada de análise de regressão em crista ou em cumeeira. Todas as análises foram realizadas com auxílio do programa Genes (Cruz, 2006).

\section{Resultados e Discussão}

Observaram-se diferenças significativas entre os genótipos para todas as características avaliadas (Tabela 1). O coeficiente de variação experimental (CVe) variou de 10,38 a 42,62\%: as características SST, APl, LFo, EPol, CFr, CPFo, DF e CFo apresentaram valores de CVe abaixo de 20\%; FFr, DCaFr, Ratio, NFrAx, NFr, NFlPed e AAT de 20 a 40\%; e PFr acima de $40 \%$.

Por sua vez, o coeficiente de variação genético (CVg) variou de 12,31 a 60,54\%, para as características CPFo e NFr, respectivamente. Os altos valores dos coeficientes de variação relatados se devem, possivelmente, à variabilidade do material genético utilizado na análise, que incluiu germoplasma ainda com alta variabilidade intra-acesso. Estudos em outras culturas demonstram que alta variabilidade dos genótipos facilita o processo de seleção de plantas superiores (Sirisena $\&$ Senanayake, 2000; Aycicek \& Yildirim, 2006; Biçer \& Şakar, 2008).

Observa-se que a amplitude dos valores das características com maior $\mathrm{CVe}$ e $\mathrm{CVg}$ é bastante elevada, como no caso do NFLPed (1,00 a 12,00); NFrAx (1,00 a 6,00); NFr (1,00 a 166,00); FFr $(1,00$ a 7,75$)$ e $\operatorname{PFr}(168,86$ a $2.326,00)$ (Tabela 1). Essas observações são importantes e revelam amplo potencial desse germoplasma para o desenvolvimento de novas variedades, uma vez que a existência de variação genética garante o sucesso das ações nos programas de melhoramento. Silva et al. (2007) observaram valores de CVe entre 1,28 e 66,25, para peso médio de frutos e número de frutos pentândricos, respectivamente; e $\mathrm{CVg}$ entre 5,39 e 124,21 para as características diâmetro do caule aos 260 dias após plantio e número de frutos pentândricos, respectivamente.

A correlação genotípica foi maior do que a fenotípica em todas as relações avaliadas (Tabela 2), o que é favorável ao processo de seleção, uma vez que 
os efeitos genéticos se sobrepõem aos ambientais na manifestação do fenótipo. Resultados semelhantes foram encontrados na análise de características relacionadas à qualidade fisiológica de sementes de maracujazeiro (Araújo et al., 2007). Além disso, verifica-se que, de modo geral, as correlações existentes entre NFre as demais variáveis forambaixas. Entretanto, observou-se correlação genética significativa e positiva entre NFr e NFrAx. Por sua vez, correlações negativas foram observadas entre NFr e CFr, NFr e DFr, NFr e Epol, NFr e PFr, e NFr e DCaFr.

Além das correlações com NFr, outras correlações positivas e significativas foram observadas entre as características $\mathrm{CPF}$ e $\mathrm{CF}$, LFo, $\mathrm{PFr}, \mathrm{DFr}, \mathrm{DCaFr}$; CFo e LFo, APl, PFr, CFr, DFr, DCaFr; LFo e APl, PFr; NFrAx e NFrPl; FFr e EPol; PFr e CFr, DFr, DCaFr; CFr e DFr, DCaFr, EPol; DFr e DCaFr, EPol; ATT e SST e SST e Ratio (Tabela 2). Também foram observadas correlações negativas e significativas entre NFrAx e CFr, DFr, DCaFr, EPol; NFrPl e PFr, CFr, DFr, DCaFr, EPol; FFr e Ratio; PFr x SST; CFr x SST; DFr e SST e Ratio e DCaFr e SST e Ratio.

Estas correlações indicam a possibilidade de se promover seleção indireta em importantes características de frutos, como peso, diâmetro e cavidade interna do fruto, pelo uso de características morfológicas de fácil mensuração, como CPFo e CFo. Já CFo e LFo apresentaram correlação positiva com APl, o que indica que a redução da largura e do comprimento das folhas induzem à redução na altura das plantas, o que é muito importante nos sistemas de produção dessa cultura, tendo em vista que plantas muito altas dificultam os tratos culturais e a colheita.

A identificação da correlação entre características de fácil mensuração e as relacionadas à produtividade e à qualidade de frutos é um dos objetivos dos programas de melhoramento, para que se possa facilitar e acelerar a seleção de plantas superiores. Neste sentido, Silva et al. (2007) sugerem que a seleção de plantas de mamoeiro com maior diâmetro do caule pode resultar em plantas mais produtivas, em virtude da alta correlação genética entre essas características $(0,84)$. O trabalho de Fraife Filho et al. (2001) corrobora esses resultados, com correlação fenotípica de 0,93 entre produtividade média anual e diâmetro de caule.

Também observou-se que frutos com maior espessura da polpa tendem a apresentar maior firmeza de fruto. Por sua vez, existe correlação negativa com o Ratio. Tal observação indica que a combinação Brix e ATT pode influenciar a firmeza de frutos, embora não se tenha observado correlação significativa com essas características de forma isolada.

Tabela 1. Resumo das análises de variância, com os coeficientes de variação experimental (CVe) e genético (CVg) e os valores mínimos e máximos das características morfológicas e agronômicas avaliadas nos genótipos de mamoeiro.

\begin{tabular}{|c|c|c|c|c|c|c|}
\hline Característica $^{(1)}$ & Quadrado médio (genótipos) & Média_erro padrão & $\mathrm{CVe}(\%)$ & CVg $(\%)$ & Mínimo & Máximo \\
\hline CPFo & $687,36^{* *}$ & $62,26 \pm 3,52$ & 16,02 & 12,31 & 18,00 & 106,00 \\
\hline CFo & $333,86^{* *}$ & $35,06 \pm 2,81$ & 16,54 & 15,63 & 14,00 & 68,00 \\
\hline LFo & $816,28 * *$ & $53,29 \pm 3,29$ & 12,14 & 16,51 & 30,00 & 85,00 \\
\hline APl & $12.527,28 * *$ & $206,25 \pm 6,43$ & 11,12 & 16,80 & 95,00 & 340,00 \\
\hline NFIPed & $26,31 * *$ & $3,82 \pm 1,45$ & 39,28 & 40,62 & 1,00 & 12,00 \\
\hline NFrAx & $3,83 * *$ & $1,50 \pm 0,88$ & 34,44 & 39,68 & 1,00 & 6,00 \\
\hline NFr & $4.720,67 * *$ & $35,22 \pm 4,98$ & 37,43 & 60,54 & 1,00 & 166,00 \\
\hline $\mathrm{FFr}$ & $9,73 * *$ & $3,20 \pm 1,01$ & 23,88 & 29,86 & 1,00 & 7,75 \\
\hline $\mathrm{PFr}$ & $87.456,60^{* *}$ & $644,84 \pm 19,73$ & 42,62 & 43,83 & 168,86 & $2.326,00$ \\
\hline $\mathrm{CFr}$ & $124,09 * *$ & $18,17 \pm 2,10$ & 15,59 & 18,75 & 9,50 & 35,00 \\
\hline $\mathrm{DFr}$ & $19,09 * *$ & $8,88 \pm 1,39$ & 16,19 & 14,69 & 4,90 & 14,90 \\
\hline $\mathrm{DCaFr}$ & $10,49 * *$ & $4,90 \pm 1,23$ & 24,04 & 19,45 & 1,70 & 11,20 \\
\hline EPol & $1,05^{* *}$ & $2,43 \pm 0,66$ & 13,10 & 12,72 & 1,10 & 3,70 \\
\hline ATT & $0,003 * *$ & $0,08 \pm 0,02$ & 27,13 & 20,13 & 0,04 & 0,59 \\
\hline SST & $29,89 * *$ & $11,98 \pm 1,46$ & 10,38 & 14,05 & 5,00 & 16,20 \\
\hline Ratio & $8.835,72 * *$ & $163,73 \pm 7,03$ & 25,31 & 16,30 & 25,90 & 360,79 \\
\hline
\end{tabular}

Pesq. agropec. bras., Brasília, v.45, n.8, p.855-862, ago. 2010 
Da mesma forma, frutos com maior comprimento, diâmetro, cavidade interna e espessura de polpa tendem a ter maior peso. Contudo, existe tendência para redução no Brix (Tabela 2). O aumento na cavidade interna pode levar à redução em SST e Ratio, o que é indesejável para o consumidor da fruta. Além disso, frutos com maior diâmetro da cavidade interna são mais suscetíveis às perdas ocorridas durante o transporte e armazenamento. Por sua vez, as correlações positivas entre SST, ATT e Ratio indicam a possibilidade de sucesso na seleção de genótipos com maior equilíbrio no sabor da fruta.

Entre todas as características avaliadas, o NFr possui a maior importância econômica, pois é um componente de produção que deve ser levado em consideração no processo de seleção de genótipos produtivos e adaptados aos diferentes sistemas de produção do mamoeiro. Entretanto, verificou-se que a maioria das variáveis explicativas estão correlacionadas entre si, o que mostra a complexidade da relação entre as características relacionadas ao NFr. Portanto, o esforço da pesquisa para o desenvolvimento de ferramentas metodológicas visando ao aumento no NFr deve ser focado no desdobramento das correlações existentes em seus efeitos diretos e indiretos, para avaliar o grau de importância de cada uma das variáveis explicativas com a principal.

Tabela 2. Estimativas dos coeficientes de correlação fenotípica $\left(\mathrm{r}_{\mathrm{f}}\right)$ e genotípica $\left(\mathrm{r}_{\mathrm{g}}\right)$ entre 16 características morfológicas e agronômicas de mamoeiro.

\begin{tabular}{|c|c|c|c|c|c|c|c|c|c|c|c|c|c|c|c|c|}
\hline Característica $^{(1)}$ & & $\mathrm{CFo}$ & LFo & $\mathrm{APl}$ & NFIPed & NFrAx & $\mathrm{NFr}$ & $\mathrm{FFr}$ & $\mathrm{PFr}$ & $\mathrm{CFr}$ & $\mathrm{DFr}$ & $\mathrm{DCaFr}$ & EPol & ATT & SST & Ratio \\
\hline \multirow[t]{2}{*}{ CPFo } & rf & $0,80 * *$ & $0,55 * *$ & 0,37 & $-0,08$ & $-0,17$ & $-0,17$ & 0,03 & $0,54 * *$ & 0,34 & $0,51 *$ & $0,58 * *$ & 0,07 & $-0,03$ & $-0,11$ & 0,07 \\
\hline & $\mathrm{rg}$ & 0,83 & 0,59 & 0,39 & $-0,11$ & $-0,18$ & $-0,19$ & 0,04 & 0,61 & 0,38 & 0,58 & 0,68 & 0,09 & $-0,01$ & $-0,13$ & 0,07 \\
\hline \multirow[t]{2}{*}{ CFo } & $\mathrm{rf}$ & & $0,66^{* *}$ & $0,44 *$ & 0,05 & $-0,02$ & $-0,11$ & $-0,06$ & $0,71 * *$ & $0,57 * *$ & $0,60 * *$ & $0,58^{* *}$ & 0,14 & $-0,14$ & $-0,26$ & $-0,06$ \\
\hline & $\mathrm{rg}$ & & 0,69 & 0,46 & 0,05 & $-0,01$ & $-0,12$ & $-0,06$ & 0,78 & 0,61 & 0,67 & 0,66 & 0,17 & $-0,17$ & $-0,29$ & $-0,09$ \\
\hline \multirow[t]{2}{*}{ LFo } & rf & & & $0,45^{*}$ & $-0,21$ & 0,27 & 0,29 & 0,13 & $0,46^{*}$ & 0,33 & 0,30 & 0,27 & $-0,11$ & $-0,14$ & $-0,20$ & 0,02 \\
\hline & $\mathrm{rg}$ & & & 0,47 & $-0,23$ & 0,29 & 0,30 & 0,14 & 0,50 & 0,34 & 0,32 & 0,30 & $-0,13$ & $-0,17$ & $-0,22$ & 0,02 \\
\hline \multirow[t]{2}{*}{$\mathrm{APl}$} & rf & & & & $-0,25$ & 0,07 & 0,29 & 0,19 & 0,19 & 0,11 & 0,10 & 0,08 & 0,06 & 0,22 & 0,25 & $-0,04$ \\
\hline & $\mathrm{rg}$ & & & & $-0,28$ & 0,07 & 0,29 & 0,20 & 0,21 & 0,13 & 0,11 & 0,09 & 0,07 & 0,27 & 0,25 & $-0,05$ \\
\hline \multirow[t]{2}{*}{ NFIPed } & rf & & & & & 0,17 & 0,06 & $-0,18$ & 0,16 & 0,11 & 0,00 & $-0,02$ & $-0,01$ & $-0,07$ & 0,02 & 0,06 \\
\hline & $\mathrm{rg}$ & & & & & 0,17 & 0,05 & $-0,21$ & 0,16 & 0,10 & 0,00 & $-0,02$ & $-0,02$ & $-0,08$ & 0,03 & 0,08 \\
\hline \multirow[t]{2}{*}{ NFrAx } & rf & & & & & & $0,84 * *$ & $-0,21$ & $-0,30$ & $-0,44 *$ & $-0,54^{*}$ & $-0,36^{*}$ & $-0,73 * *$ & $-0,07$ & 0,22 & 0,31 \\
\hline & $\mathrm{rg}$ & & & & & & 0,86 & $-0,22$ & $-0,33$ & $-0,47$ & $-0,59$ & $-0,41$ & $-0,80$ & $-0,08$ & 0,24 & 0,36 \\
\hline \multirow[t]{2}{*}{$\mathrm{NFr}$} & rf & & & & & & & $-0,08$ & $-0,43^{*}$ & $-0,61 * *$ & $-0,62 * *$ & $-0,51^{*}$ & $-0,58 * *$ & 0,20 & 0,36 & 0,23 \\
\hline & $\mathrm{rg}$ & & & & & & & $-0,09$ & $-0,46$ & $-0,63$ & $-0,67$ & $-0,55$ & $-0,62$ & 0,25 & 0,37 & 0,25 \\
\hline \multirow[t]{2}{*}{ FFr } & rf & & & & & & & & 0,32 & 0,29 & 0,21 & 0,15 & $0,40 *$ & 0,24 & $-0,17$ & $-0,53 * *$ \\
\hline & $\mathrm{rg}$ & & & & & & & & 0,34 & 0,30 & 0,22 & 0,16 & 0,42 & 0,30 & $-0,18$ & $-0,62$ \\
\hline \multirow[t]{2}{*}{$\mathrm{PFr}$} & rf & & & & & & & & & $0,89 * *$ & $0,86^{* *}$ & $0,75^{* *}$ & $0,53^{*}$ & $-0,15$ & $-0,49^{*}$ & $-0,40$ \\
\hline & $\mathrm{rg}$ & & & & & & & & & 0,90 & 0,87 & 0,77 & 0,53 & $-0,20$ & $-0,51$ & $-0,43$ \\
\hline \multirow[t]{2}{*}{$\mathrm{CFr}$} & rf & & & & & & & & & & $0,84 * *$ & $0,68^{* *}$ & $0,60 * *$ & $-0,18$ & $-0,46^{*}$ & $-0,40$ \\
\hline & $\mathrm{rg}$ & & & & & & & & & & 0,87 & 0,72 & 0,61 & $-0,25$ & $-0,48$ & $-0,42$ \\
\hline \multirow[t]{2}{*}{$\mathrm{DFr}$} & rf & & & & & & & & & & & $0,92 * *$ & $0,56^{* *}$ & $-0,14$ & $-0,55^{* *}$ & $*-0,46^{*}$ \\
\hline & $\mathrm{rg}$ & & & & & & & & & & & 0,93 & 0,57 & $-0,20$ & $-0,57$ & $-0,49$ \\
\hline \multirow[t]{2}{*}{$\mathrm{DCaFr}$} & rf & & & & & & & & & & & & 0,28 & $-0,09$ & $-0,47 *$ & $-0,43^{*}$ \\
\hline & $\mathrm{rg}$ & & & & & & & & & & & & 0,26 & $-0,13$ & $-0,49$ & $-0,46$ \\
\hline \multirow[t]{2}{*}{ EPol } & $\mathrm{rf}$ & & & & & & & & & & & & & 0,11 & $-0,32$ & $-0,44$ \\
\hline & $\mathrm{rg}$ & & & & & & & & & & & & & 0,12 & $-0,33$ & $-0,47$ \\
\hline \multirow[t]{2}{*}{ ATT } & rf & & & & & & & & & & & & & & $0,48^{* *}$ & $*-0,38$ \\
\hline & $\mathrm{rg}$ & & & & & & & & & & & & & & 0,60 & $-0,32$ \\
\hline \multirow[t]{2}{*}{ SST } & rf & & & & & & & & & & & & & & & $0,50^{*}$ \\
\hline & $\mathrm{rg}$ & & & & & & & & & & & & & & & 0,55 \\
\hline
\end{tabular}

${ }^{(1)} \mathrm{CPFo}$, comprimento do pecíolo da folha (cm); CFo, comprimento da folha madura (cm); LFo, largura da folha madura (cm); $\mathrm{APl}$, altura da planta (cm); NFIPed, número de flores por pedúnculo; $\mathrm{NfrAx}$, número de frutos por axila; $\mathrm{NFr}$, número de frutos por planta; $\mathrm{FFr}$, firmeza do fruto (libras $\left.\mathrm{cm}^{-2}\right)$; $\mathrm{PFr}$, massa do fruto (g); CFr, comprimento do fruto (cm); DFr, diâmetro do fruto (cm); DCaFr, diâmetro da cavidade do fruto (cm); Epol, espessura da polpa (cm); ATT, acidez total titulável (g de ácido cítrico por $100 \mathrm{~mL}$ ); SST, sólidos solúveis totais, em ${ }^{\circ}$ Brix; e Ratio, razão entre ATT e SST. * e **Significativo a 5 e $1 \%$ de probabilidade, pelo teste $t$. 


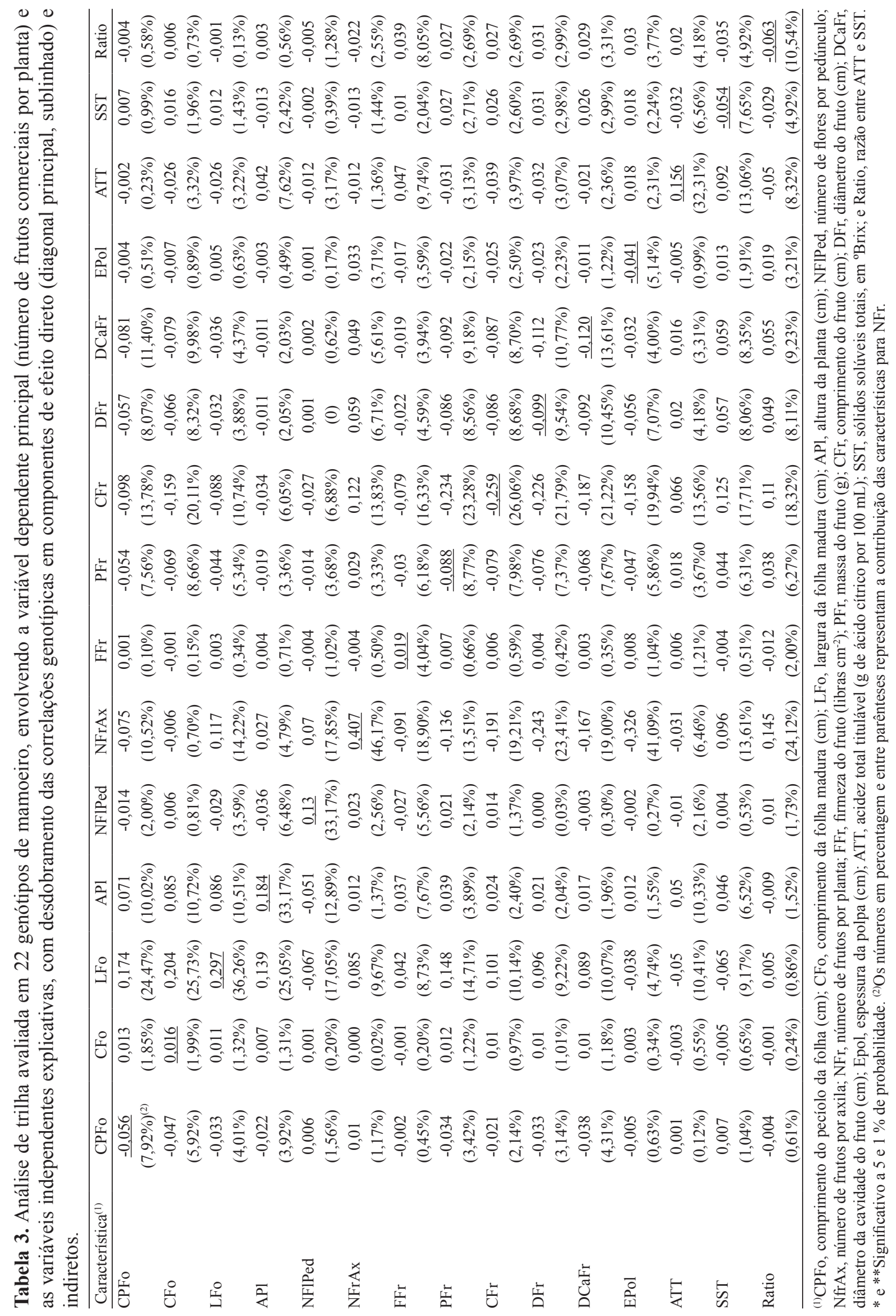


A análise dos coeficientes de trilha da característica NFr como variável dependente encontra-se na Tabela 3. $\mathrm{O}$ coeficiente de determinação $\left(\mathrm{R}^{2}\right)$ dessa análise foi de 0,87 e o efeito da variável residual foi de 0,25 , o que indica o bom ajuste do modelo na explicação dos efeitos genéticos relacionados à variável em análise.

Foram observados efeitos diretos e positivos das características CFo, LFo, APl, NFIPed, NFrAx, FFr e ATT sobre a variável dependente, embora CFo e FFr tenham apresentado pequeno efeito direto, abaixo de $26 \%$ (Tabela 3). A característica NFrAx apresentou o maior efeito direto para aumento de NFr $(46,17 \%$ dos efeitos), com efeito indireto e positivo via CFr. Esses dados corroboram o efeito das correlações genéticas simples de NFrAx e NFr e CFr e NFr (Tabela 2). Embora o efeito indireto de CFr seja baixo (13,83\%), diferentemente da correlação simples, a análise de trilha revelou efeitos positivos em relação à NFr (Tabela 3).

No melhoramento, é importante identificar, entre as variáveis de alta correlação com a variável básica, aquelas de maior efeito direto em sentido favorável à seleção, de tal forma que a resposta correlacionada por meio da seleção indireta seja eficiente (Cruz et al., 2004). Neste trabalho, a variável NFrAx apresentou esse atributo. Entretanto, a mensuração dessa característica deve ser realizada durante a formação de frutos e, neste caso, a contagem direta da característica NFr seria mais viável.

Apesar de não se ter detectado efeito significativo na análise de correlação simples (Tabela 2), as características LFo, APl, NFlPed e ATT apresentaram efeitos diretos positivos e significativos para a seleção de plantas com maior número de frutos: 36,$26 ; 33,17$; 33,17 e $32,31 \%$, respectivamente (Tabela 3). Isso provavelmente deve-se à existência de diversos efeitos indiretos negativos que mascaram o efeito dessas características na expressão da variável NFr. Portanto, há possibilidade de se utilizar características que se manifestam antes da produção dos frutos, como LFo, APl e NFIPed, pra a seleção indireta para NFr.

Também foram identificados efeitos diretos $\mathrm{e}$ negativos das características $\mathrm{CPFo}, \mathrm{PFr}, \mathrm{CFr}, \mathrm{DFr}$, DCaFr, EPol, SST e Ratio sobre NFr (Tabela 3). Entretanto, apesar dos elevados valores de correlação genotípica com NFr, as características PFr, DFr, $\mathrm{DCaFr}$ e EPol apresentaram pequenos efeitos diretos sobre essa característica, inferiores até mesmo aos respectivos valores de efeito da variável residual. Esse fato reduz a importância dessas características em relação à variável CFr (26,06\%). Os maiores efeitos indiretos foram observados para CFr via LFo $(10,14 \%)$ e NFrAx $(13,51 \%)$.

De acordo com Cruz et al. (2004), variáveis com alta correlação favorável, mas com baixo efeito direto, indicam que a seleção truncada na variável auxiliar pode proporcionar ganhos satisfatórios na variável principal. Neste caso, a melhor estratégia é a seleção simultânea de variáveis, com ênfase também naquelas cujos efeitos indiretos sejam significativos.

Verificou-se que o efeito indireto de PFr via LFo e NFrAx; DFr via NFrAx e CFr; DCaFr via LFo, NFrAx, CFr e DFr; EPol via NFrAx e CFr foram mais importantes do que o próprio efeito direto dessas sobre NFr (Tabela 3). Portanto, essas características não são indicadas para uso nos programas de melhoramento visando à seleção dirigida para aumento no número de frutos comerciais por planta. De modo geral, observaram-se altas contribuições indiretas das características LFo, NFrAx e CFr, o que demonstra seu potencial para uso no processo de seleção para NFr.

\section{Conclusões}

1. A alta variabilidade genética observada entre os acessos do banco de germoplasma do mamoeiro permite a seleção de genótipos com tipos diferenciados de plantas e frutos, para uso comercial ou em programas de melhoramento genético.

2. As estimativas das correlações genotípicas foram maiores do que as fenotípicas, o que indica que há maior influência do genótipo sobre os efeitos ambientais e boas possibilidades de ganhos genéticos com a seleção de plantas.

3. O número de frutos por axila e o comprimento de fruto possuem maior efeito direto sobre número de frutos por planta, o que as torna mais indicadas para aumentar as chances de sucesso na seleção de genótipos de mamoeiro mais produtivos.

4. As características número de frutos por axila, largura da folha, altura da planta, número de flores por pedúnculo, comprimento de fruto e acidez são as principais determinantes das variações no número de frutos por planta.

5. Entre as características que apresentam facilidade de mensuração e que se manifestam antes da produção 
de frutos, altura da planta, número de flores por pedúnculo e largura de folhas demonstraram alto potencial para uso nos programas de melhoramento genético visando à seleção indireta de número de frutos por planta, sobretudo pelos seus efeitos diretos.

\section{Agradecimentos}

Ao Conselho de Desenvolvimento Científico e Tecnológico e Fundação de Amparo à Pesquisa do Estado da Bahia, pelo apoio.

\section{Referências}

ALMEIDA, A.H.B. Heterose e correlações em plantas branquícas e normais de jerimum caboclo (Cucurbita maxima Duchesne). 1988. 95p. Dissertação (Mestrado) - Universidade Federal de Viçosa, Viçosa.

ARAÚJO, E.C. de; DAHER, R.F.; SILVA, R.F. da; PIO VIANA, A. Path analysis for physiological traits that influence seed germination of Passiflora edulis f. flavicarpa Deg. Crop Breeding and Applied Biotechnology, v.7, p.148-154, 2007.

ASSOCIAÇÃO BRASILEIRA DOS EXPORTADORES DE PAPAYA. Brapex - Associação Brasileira dos Exportadores de Papaya [home page]. Disponível em: <http://www.brapex.net/ index_1024.asp>. Acesso em: 27 dez. 2006.

AYCICEK, M.; YILDIRIM, T. Path coefficient analysis of yield and yield components in bread wheat (Triticum aestivum L.) genotypes. Pakistan Journal of Botany, v.38, p.417-424, 2006.

BIÇER, B.T.; ŞAKAR, D. Heritability and path analysis of some economical characteristics in lentil. Journal of Central European Agriculture, v.9, p.191-196, 2008.

CARVALHO, C.G.P. de; ARIAS, C.A.A.; TOLEDO, J.F.F. de; OLIVEIRA, M.F. de; VELLO, N.A. Correlações e análise de trilha em linhagens de soja semeadas em diferentes épocas. Pesquisa Agropecuária Brasileira, v.37, p.311-320, 2002.

CARVALHO, C.G.P. de; OLIVEIRA, V.R.; CRUZ, C.D.; CASALI, V.W.D. Análise de trilha sob multicolinearidade em pimentão. Pesquisa Agropecuária Brasileira, v.34, p.603-613, 1999.

CARVALHO, F.I.F.; LORENCETTI, C.; BENIN, G. Estimativas e implicações da correlação no melhoramento vegetal. Pelotas: UFPel, 2004. 142p.

CARVALHO, S.P. de. Métodos alternativos de estimação de coeficientes de trilha e índices de seleção, sob multicolinearidade. Viçosa: UFV, 1995. 163p.
CHAUDHARY, D.; SRIVASTA, D.P.; GHOSH,A.K.; SEETHARA, $\mathrm{R}$. Genetic variability and correlation for yield components in rice. Indian Journal of Agricultural Sciences, v.43, p.181-184, 1973.

CRUZ, C.D. Programa Genes: biometria. Viçosa: UFV, 2006 a. 382p.

CRUZ, C.D.; REGAZZI, A.J.; CARNEIRO, P.C.S. Modelos biométricos aplicados ao melhoramento genético. 3.ed. Viçosa: UFV, 2004. 480p.

FRAIFE FILHO, G. de A.; DANTAS, J.L.L.; LEITE, J.B.V.; OLIVEIRA, J.R.P. Avaliação de variedades de mamoeiro no Extremo Sul da Bahia. Magistra, v.13, p.37-41, 2001.

MARTINS, D. dos S.; COSTA, A. de F.S. da. A cultura do mamoeiro: tecnologias de produção. Vitória: Incaper, 2003. 497p.

MONTGOMERY, D.C.; PECK, E.A. Introduction to linear regression analysis. New York: Wiley, 1982. 504p.

NEGREIROS, J.R. da S.; ÁLVARES, V. de S.; BRUCKNER, C.H.; MORGADO, M.A.D.O.; CRUZ, C.D. Relação entre características físicas e o rendimento de polpa de maracujá-amarelo. Revista Brasileira de Fruticultura, v.29, p.546-549, 2007.

OKUT, H.; AKCA, Y. Study to determine the causal relations between fruit weight and certain important fruit characteristics with using a path analysis. Acta Horticulturae, n.384, p.97-102, 1995.

REYNOLDS, A.G.; WARDLE, D.A. Yield component path analysis of okanagan riesling vines conventionally pruned or subjected to simulated mechanical pruning. American Journal of Enology and Viticulture, v.44, p.173-179, 1993.

SANTOS, J.; VENCOVSKY, R. Correlação fenotípica e genética entre alguns caracteres agronômicos do feijoeiro (Phaseolus vulgaris L.). Ciência e Prática, v.10, p.265-272, 1986.

SCHUSTER, I. Correlações, coeficientes de trilha, composição de gluteninas e qualidade do trigo para panificação. Viçosa: UFV, 1996. 98p.

SILVA, F.F. da; PEREIRA, M.G.; RAMOS, H.C.C.; DAMASCENO JUNIOR, P.C.; PEREIRA, T.N.S.; IDE, C.D. Genotypic correlations of morpho-agronomic traits in papaya and implications for genetic breeding. Crop Breeding and Applied Biotechnology, v.7, p.345-352, 2007

SIRISENA, J.A.; SENANAYAKE, S.G.J.N. Estimation of variability parameters within 'Mysore' banana clones and their implication for crop improvement. Scientia Horticulturae, v.84, p.49-66, 2000.

WRIGHT, S. Correlation and causation. Journal of Agricultural Research, v.20, p.557-585, 1921.

WRIGHT, S. The theory of path coefficients a reply to niles's criticism. Genetics, v.8, p.239-255, 1923.

Recebido em 14 de abril de 2010 e aprovado em 16 de julho de 2010 